
IMMEDIATE AND PROFOUND HEPATOCELLULAR DAMAGE AND INFLAMMATION IN HUMANS

doi:10.1136/gutjnl-2011-300857a.32

${ }^{1} \mathrm{M}$ A J van den Broek, ${ }^{2} \mathrm{R}$ Shiri-Sverdlov, ${ }^{1} \mathrm{~J} \mathrm{~J}$ W Schreurs, ${ }^{1} \mathrm{~J}$ G Bloemen, ${ }^{2} \mathrm{~V}$ Bieghs, ${ }^{1}$ W A Buurman, ${ }^{1} \mathrm{C}$ H C Dejong, 'S W M Olde Damink. 'Department of General Surgery, Maastricht University, Maastricht, the Netherlands; ${ }^{2}$ Department of Molecular Genetics, Maastricht University, Maastricht, the Netherlands

Introduction In a recent study we coincidentally showed that mobilisation of the liver was a major cause of liver surgery-induced damage. The magnitude of and mechanisms by which this damage occurs are unknown.

Aim (A) To determine the relative contribution of mobilisation during liver surgery to liver damage and (B) To examine whether there is an association between mobilisation-induced liver damage and liver inflammation.

Method Consecutive patients undergoing liver surgery requiring full mobilisation of the right hemi-liver were included. Plasma samples and liver biopsies were obtained immediately after induction, prior to and directly after liver mobilisation, and after liver transection. Liver Fatty Acid Binding Protein (L-FABP) and alanine aminotranferase (ALAT) were analysed as markers of hepatocyte injury. Specimens were stained by immunohistochemistry for myeloperoxidase (MPO), human neutrophil peptide (HNP), and caspase-3mediated cleavage generated neo-epitope of CK18 (M30). Gene expression of interleukin (IL) $1 \beta, 6$ and 8, and intercellular adhesion molecule (ICAM) were analysed by q-RT-PCR

Results Nineteen patients were included (11M/8F, median age 64 years [30-79]) who underwent major liver surgery. L-FABP levels increased significantly during liver mobilisation (from $91.7 \mathrm{ng} / \mathrm{ml}$ $[11.4-2212.5 \mathrm{ng} / \mathrm{ml}]$ to $1014.4 \mathrm{ng} / \mathrm{ml} \quad[141.4-8986.1 \mathrm{ng} / \mathrm{ml}]$, $\mathrm{p}<0.001)$ and did not increase significantly thereafter $(1315.2 \mathrm{ng} / \mathrm{ml}$ $[67.0-20099.2 \mathrm{ng} / \mathrm{ml}], \mathrm{p}=0.75)$. L-FABP levels after $\geq 60 \mathrm{~min}$ mobilisation time were significantly higher when compared to $\geq 60$ min mobilisation $(1679.7 \mathrm{ng} / \mathrm{ml}$ vs $645.9 \mathrm{ng} / \mathrm{ml}, \mathrm{p}=0.04)$. ALAT levels increased significantly from $26 \mathrm{IU} / \mathrm{l}$ [13-147] before to $130 \mathrm{IU} / 1$ [74-813] after liver mobilisation and to $275 \mathrm{IU} / 1$ [13-1352] after transection (all $\mathrm{p}<0.05$ ). Liver mobilisation increased the numbers of positive cells in staining for MPO $(p=0.0007)$, HNP $(p=0.03)$, and M30 $(p=0.01)$, whereas transaction led to no further increase thereafter. Liver mobilisation increased the gene expression of IL1b $(p=0.01)$, IL- $6(p=0.08)$, IL-8 $(p=0.02)$ and ICAM $(p=0.007)$. Expression increases ranged from 2.8-fold in ICAM to 130-fold in IL-6. After transection, mRNA levels increased even further in IL-6 $(p=0.004)$, IL-8 $(p=0.0004)$ and ICAM $(p=0.02)$, but not IL1b ( $\mathrm{p}=0.32)$.

Conclusion Mobilisation of the liver during surgery induces profound hepatocellular damage and inflammation, which is associated with activation and/or infiltration of immune cells. Given the short half-life of L-FABP (14 min), hepatocyte damage predominantly occurred during mobilisation of the liver and not during transaction. These data produce insight into the mechanisms of mobilisation-induced liver damage, and provide indications for designing interventions aiming at prevention of surgery-induced liver damage in the future.

\section{P33 SERUM TOTAL CORTISOL AND PLASMA FREE CORTISOL RESPONSE AFTER LOW DOSE SHORT SYNACTHEN TEST IN STABLE CIRRHOSIS}

doi:10.1136/gutjnl-2011-300857a.33

${ }^{1} \mathrm{G}$ Fede, ${ }^{1} \mathrm{~L}$ Spadaro, ${ }^{1} \mathrm{~T}$ Tomaselli, ${ }^{1} \mathrm{G}$ Privitera, ${ }^{1} \mathrm{R}$ Scicali, ${ }^{2} \mathrm{P}$ Vasianopoulou, ${ }^{3} \mathrm{~N}$ Martin, ${ }^{3} \mathrm{M}$ Thomas, ${ }^{1} \mathrm{~F}$ Purrello, ${ }^{2} \mathrm{~A}$ K Burroughs. ${ }^{1}$ Department of Internal Medicine,
University of Catania, Garibaldi Hospital, Catania, Italy; ${ }^{2}$ The Royal Free Sheila Sherlock Liver Centre and University Department of Surgery-University College London and Royal Free Hospital, London, UK; ${ }^{3}$ Department of Clinical Biochemistry-Royal Free Hospital, London, UK

Introduction Adrenal Insufficiency (AI) defined by low dose short synacthen test (LDSST) in stable cirrhosis is frequent using serum total cortisol (TC). However no published data exist on directly measured plasma free cortisol (FC) after LDSST.

Aim We prospectively assessed adrenal insufficiency defined by LDSST in stable cirrhosis using TC and FC.

Method Patients with stable cirrhosis without shock and/or sepsis were prospectively studied using the LDSST. AI was defined by a peak-TC $<494 \mathrm{mmol} / \mathrm{l}$ (Criterion 1) and a peak-FC $<33 \mathrm{nmol} /$ (Criterion 2) $30 \mathrm{~min}$ after injection of $1 \mu \mathrm{g}$ of tetracosactrin (Synacthen).

Results 78 consecutive patients with cirrhosis were studied (Viral 16, Alcoholic: 41; other: 21). Basal TC (365 $\pm 192 \mathrm{mmol} / \mathrm{l})$ and peak TC $(571 \pm 216 \mathrm{mmol} / \mathrm{l})$ were significantly related to basal FC $(26 \pm 24 \mathrm{nmol} / \mathrm{l})$ and peak FC $(53 \pm 34 \mathrm{nmol} / \mathrm{l})$ respectively: for baseline values $R=0.78, p<0.001$, and for peak values $R=0.70$, $p<0.05$. Similar results were found considering only patients with hypoalbuminemia (albumin $<25 \mathrm{~g} / 1,7$ patients): for basal value $\mathrm{R}=0.88, \mathrm{p}<0.001$; for peak value $\mathrm{R}=0.89, \mathrm{p}<0.05$. Prevalence of $\mathrm{AI}$ was $35 \%(27 / 78)$ using total cortisol (Criterion 1$)$ and $28 \%(22 / 78)$ using free cortisol (Criterion 2). There was agreement between total cortisol (Criterion 1) and free cortisol (Criterion 2) in 63 tests, in 10 AI was diagnosed only according TC (Criterion 1), and in 5 only according FC (Criterion 2 ): $\kappa$-coefficient $0.56, \mathrm{p}<0.05$. In the group with discordant tests patients had more advanced liver disease (Child score: $9.17 \pm 2.2$ vs $7.66 \pm 1.9, p=0.03$ ), lower basal TC $(237 \pm 104$ vs $395 \pm 196 \mathrm{mmol} / \mathrm{l}, \mathrm{p}=0.03)$, and peak TC $(464 \pm 121 \mathrm{vs}$ $597 \pm 226 \mathrm{mmol} / \mathrm{l}, \mathrm{p}=0.009$ ).

Conclusion AI defined by LDSST is frequent in stable patients with cirrhosis, using both total cortisol and free cortisol criteria. However in patients with more advanced liver disease and/or low total cortisol level, discrepancy exists between the rates of diagnosis of AI using the total and free cortisol criteria. Thus in these patients AI should be confirmed by free cortisol measurement.

\section{P34 NO EFFECT OF TENOFOVIR OR ENTECAVIR ON VITAMIN D LEVELS IN CHRONIC HEPATITIS B MONO-INFECTED PATIENTS. SINGLE CENTRE 'REAL LIFE' COHORT EXPERIENCE}

doi:10.1136/gutjn-2011-300857a.34

${ }^{1} \mathrm{H}-\mathrm{L}$ Nguyen, ${ }^{1} \mathrm{I}$ Carey, ${ }^{1} \mathrm{M}$ A B Al-Freah, ${ }^{1} \mathrm{D}$ Joe, ${ }^{2} \mathrm{R}$ Sherwood, ${ }^{1} \mathrm{~S}$ Knighton, ${ }^{1} \mathrm{P}$ Harrison, ${ }^{1} \mathrm{~A}$ Suddle, ${ }^{1} \mathrm{~K}$ Agarwal. ${ }^{1}$ Institute of Liver Studies, King's College Hospital, London, UK; ${ }^{2}$ Clinical Biochemistry, King's College Hospital, London, UK

Introduction Increasing evidence confirms the pivotal role of Vitamin $\mathrm{D}$ in immunomodulatory and metabolic processes. Vitamin D deficiency has an impact on long-term health outcomes. Data from HIV populations demonstrate negative effects of Tenofovir (TDF) on vitamin $\mathrm{D}(\mathrm{VD})$ levels and bone mineral density.

Aim To determine the impact of TDF on VD levels, with calcium and phosphate in patients mono-infected with HBV.

Method Retrospective study of all patients treated with TDF. VD $(\mu \mathrm{g} / \mathrm{l})$, phosphate $\left(\mathrm{PO}_{4}, \mathrm{mmol} / \mathrm{l}\right)$, calcium $\left(\mathrm{Ca}^{2+}, \mathrm{mmol} / \mathrm{l}\right)$ and alkaline phosphatase (ALP, IU/l) were checked at baseline, and 12 months after TDF or Entecavir (ETV) exposure.

Results Patients with complete data were analysed ( $n=313), 22$ patients were excluded because of combination treatment TDF and 


\section{Abstract P34 Table 1}

\begin{tabular}{llccl}
\hline \multirow{2}{*}{ Category } & Variables & TFV (n=142) & ETV (n=75) & p Value \\
\hline Demographic & Age & $41(19-80)$ & $42(19-76)$ & NS \\
& Gender: M (\%) & $143(67.5)$ & $62(80.5)$ & $<0.05$ \\
& African-Caribbean (\%) & $100(47.2)$ & $43(55.8)$ & NS \\
Clinical & Liver transplant (\%) & $9(4.3)$ & $0(0)$ & NS \\
& Hepatocellular & $14(6.8)$ & $0(0)$ & 0.014 \\
& carcinoma (\%) & & & \\
& Bilirubin & $11(3-148)$ & $12(4-37)$ & NS \\
& AST & $29(12-215)$ & $40(20-328)$ & $<0.001$ \\
VD and & INR & $1.05(0.88-1.86)$ & $1.05(0.94-2.47)$ & NS \\
Bone & Blbumin & $44(24-52)$ & $44(29-50)$ & NS \\
markers & Baseline Vitamin D (g/l) & $14.2(0-49)$ & $16.1(6-50)$ & NS \\
& Baseline Calcium mmol/l & $2.24(2.01-2.41)$ & $2.25(2.08-2.4)$ & NS \\
& 12 months Calcium mmol/l & $2.23(2.08-2.35)$ & $2.23(2.10-2.37)$ & NS \\
& Baseline Phosphate mmol// & $0.99(0.38-1.71)$ & $0.91(0.58-1.27)$ & 0.011 \\
& 12 months & $1.06(0.43-1.43)$ & $0.96(0.55-1.43)$ & 0.017 \\
& Phosphate mmol/l & & & \\
& Baseline ALP IU/I & $72(39-325)$ & $70(42-229)$ & NS \\
& 12 months ALP IU/l & $75(42-769)$ & $68(36-168)$ & 0.004 \\
\hline
\end{tabular}

ETV. There were 212 patients (73\%) treated with TDF and 79 (27\%) treated with ETV. Median age was 42 years (19-80); 266 (72\%) were Male. African-Caribbeans represented $49 \%$ and South East Asians represented $24 \%$. There were 14 patients (5\%) with hepatocellular carcinoma (HCC) and 9 (3.1) patients post liver transplantation. Median AST was 30 (12-328), Bilirubin 11 (3-148), INR 1.05 (0.88-2.47) and albumin 44 (24-73). Prevalence of VD deficiency $(\mathrm{VD}<22 \mu \mathrm{g} / \mathrm{l})$ in patients who had VD checked at baseline $(\mathrm{n}=192)$ was $81.3 \%$. Distribution of VD deficiency was not significantly different based on ethnicity $(p=N S)$, however, VD deficiency was more prevalent among men ( $85 \%$ vs $72 \%, p=0.034)$. $70 / 212$ (33\%) of those treated with TDF had VD supplements compared to $4 / 79$ $(5.1 \%)$ of ETV group, $p<0.001$. These patients were excluded from paired comparisons of VD over 12 months after TDF or ETV exposure.

In TDF treated patients, median VD was $14.2(0-49)$ at baseline and $14.7(0-41) 12$ months of therapy, $p=N S$. There was no significant difference in median $\mathrm{Ca}^{2+}(2.24$ vs $2.23, \mathrm{p}=\mathrm{NS}), \mathrm{PO}_{4}$ ( 0.99 vs $1.04, p=N S)$, however, ALP significantly increased after 12 months of TFV treatment $(71.5$ vs $75, p<0.05)$. Patients treated with ETV monotherapy, median VD at baseline was 16.1 (6-50) and after 12 months of ETV treatment was 13.6 (4.2-43.5), $\mathrm{p}=\mathrm{NS}$. No significant difference was found between baseline and 12 months $\mathrm{Ca}^{2+}$ (2.25 vs 2.23), $\mathrm{PO}_{4}$ (0.91 vs 0.96$)$ or ALP (70 vs 68), $\mathrm{p}=\mathrm{NS}$ for all comparisons. Abstract P34 table 1 demonstrate demographic, clinical and bone markers of TDF and ETV treated patients at baseline and after 12 months of therapy. TDF group had significantly lower male prevalance and AST, but a significantly increased proportion of patients with HCC, baseline and 12 months $\mathrm{PO}_{4}$ and ALP at 12 months.

Conclusion VD deficiency is highly prevalent in this cohort of CHB patients and should be appropriately identified and treated. TDF and ETV treatment had no significant effect on VD levels after 12 months of treatment in this cohort. However, there was significant rise in $\mathrm{PO}_{4}$ and ALP after 12 months of TDF treatment; which may infer indirect longer effects on bone metabolism.

\section{P35 LONG-TERM REMISSION IS ACHIEVABLE IN AUTOIMMUNE HEPATITIS USING TACROLIMUS OR MYCOPHENOLATE MOFETIL AND RESULTS IN REGRESSION OF FIBROSIS}

doi:10.1136/gutjnl-2011-300857a.35

R Scott, J White, G Atwal, N Taylor, G Mortimore, J Freeman, A Lawson, A Austin. Royal Derby Hospital

Introduction $10-20 \%$ of patients do not respond to conventional treatment of autoimmune hepatitis, or are intolerant of azathioprine. There is no established second line treatment. Experience with transplant immunosuppressive agents such as Tacrolimus (TAC) and mycophenolate mofetil (MMF) is limited to small numbers and short-term follow-up.

Aim To describe the progress of all patients who had failed conventional therapy and were treated with second line agents with at least 12-month follow-up.

Method An audit of patients identified who received second line agents for at least 12 months on maintenance dose $<10 \mathrm{mg}$ prednisolone. Patient records were reviewed and treatment endpoints based on aminotransferase changes defined as; Complete response (CR) - sustained normalisation for at least 12 months, partial response (PR) - improvement by $>50 \%$ but not always normal over a 12-month period. Where applicable, interval histology was reviewed by a single pathologist to assess ISHAK fibrosis scores at the start and at least 18 months after commencing second line agents.

Results A total of 26 patients were identified. 9 were treated with TAC for a median 81 months (21-137), 16 with MMF for a median 81 months $(30-114)$ and one on a combination of TAC and MMF. Median age is $56(28-68)$ and $64(40-79)$ respectively. The median dose of TAC is $3.5 \mathrm{mg} /$ day $(1-6)$ and MMF $1 \mathrm{~g} /$ day $(1-2)$. All patients on TAC achieved CR. Two patients discontinued treatment; one renal impairment and one rationalising treatment after 27 months CR. 11/16 patients on MMF achieved CR, 5/16 achieved PR. Five patients no longer take MMF; two due to toxicity (recurrent chest infections at 60 months, GI disturbance at 78 months), one successfully withdrew treatment after 39 months CR, one switched to TAC as a treatment failure of MMF after 103 months and one was withdrawn after a diagnosis of larynx SCC. The combination patient achieved $C R$ and has received 50 months dual treatment with confirmed histological remission. Four patients on TAC and five on MMF had interval biopsies. $3 / 4$ patients on TAC (median 87 months) exhibited stable or reduced grades of fibrosis compared to $2 / 5$ patients on MMF (median 101 months).

Conclusion Effective long-term maintenance of remission at 10 years is achievable on MMF and TAC in the absence of significant toxicity.

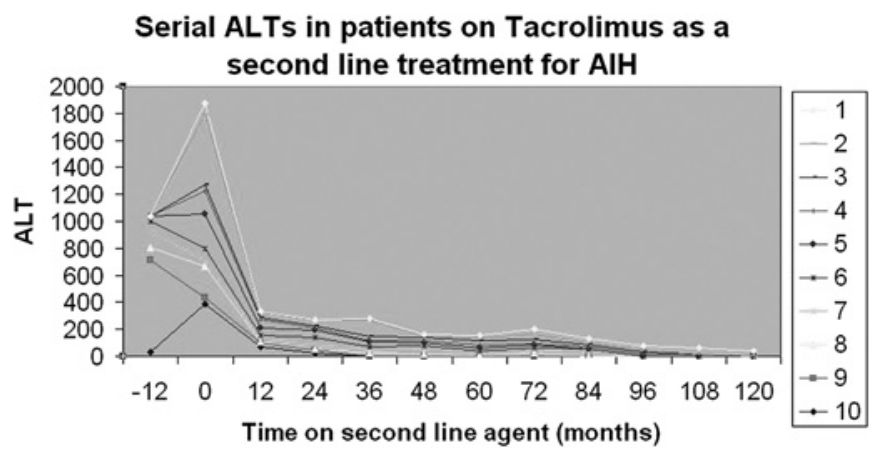

Abstract P35 Figure 1 Serial ALTs in patients on TAC as a second line treatment for AlH. 